\title{
PROPIEDADES PSICOMÉTRICAS DEL INVENTARIO PARA LA CONSTRUCCIÓN DEL CONTEXTO DE INTERVENCIÓN EN EL TRABAJO SOCIAL DE CASOS CCIP - VERSIÓN II

\author{
Psychometric properties in the Inventory for the construction of the \\ intervention context in Social Casework CCIP - 2nd version
}

Josefa Cardona Cardona ${ }^{1}$, Margalida Gili Planas², José F. Campos Vidal ${ }^{3}$

Resumen

Objetivo: Este trabajo tiene como objetivo analizar las características psicométricas del Inventario para el análisis de la relación de ayuda entre el Trabajador Social y el Cliente durante la fase de estudio y evaluación de la situación problema (Cardona y Campos, 2009). El instrumento está diseñado para conocer cuáles son los criterios de intervención que los trabajadores sociales ponen preferentemente en práctica para determinar, junto a sus clientes, cuál será el contexto de intervención para el cambio. El trabajo busca depurar el instrumento atendiendo a criterios de consistencia interna y presentar una segunda versión del mismo.

Material y métodos: La muestra, no probabilística e intencional, estuvo compuesta por el total de trabajadores sociales ubicados en proyectos de atención directa individual y familiar. Participaron un total de 115 profesionales de los cuales 9,5\% eran hombres y el 90,5\% mujeres. Respondieron al cuestionario 84 (73\%) profesionales y 31 personas rechazaron la invitación a participar o no pudieron hacerlo.

El instrumento inicial, constituido por 137 ítems, buscaba identificar y valorar los criterios de intervención más utilizados a la hora de determinar el contexto de intervención. El instrumento fue sometido a revisión de expertos para determinar su validez lógica y sus contenidos. Posteriormente, se procedió a una depuración del instrumento, se realizó un análisis exploratorio de componentes principales y un análisis factorial con rotación varimax y se determinó el coeficiente de fiabilidad (alfa de Cronbach).

Resultados y conclusiones: El banco de 137 ítems quedó reducido a 75. La solución factorial final mostró que el cuestionario estaba constituido por una única dimensión, en consonancia con la lógica procesual del instrumento. El coeficiente de consistencia interna del cuestionario reflejó un alfa de Cronbach de 0.97. Ello nos indica que se trata de un inventario cuyo contenido pone de relieve una robusta consistencia interna. La versión revisada del instrumento permite conocer los procesos implicados y aplicados en la formulación de contextos de intervención, y sirve de guía para la supervisión, el entrenamiento y la formación para la práctica del Trabajo Social de Casos.

Palabras clave: inventario, trabajo social de casos, contexto de intervención, relación de ayuda, propiedades psicométricas
Abstract

Objective: This paper aims at analysing psychometric properties in the Inventory for the analysis of the relation of help between the Social Worker and the Client during the phase of study and evaluation of the analysis of the problem situation (Cardona and Campos, 2009). This instrument is designed in order to find out the intervention criteria that social workers typically put into practice so that, together with their clients, they can determine what the intervention context for change will be. This research refines the instrument by paying particular attention to the internal consistency criteria and provides a second version of such instrument.

Material and methods: the intentional non-probabilistic sample consists of the total number of social workers who work on projects involving direct contact with the individual or family. A total number of 115 individuals took part, of which $9.5 \%$ were men and $90.5 \%$ were women. A number of 84 (73\%) professionals answered the questionnaire, whilst 31 professionals refused to take part or were unable to do it.

The initial instrument, made up of 137 ítems, aimed at identifying and assessing the intervention criteria which were most frequently applied when determining the intervention context. The instrument was revised by experts in order to determine its logical validity and its contents. After that, the instrument was refined by performing an exploratory analysis of its principal components and a factor analysis with varimax rotation, and the reliability coefficient was determined (Cronbach's alpha).

Results and conclusions: the list of 137 items was reduced to 75 . The final factorial solution showed that the questionnaire was formed by a single dimension, along with the process logic of the instrument. The coefficient of internal consistency obtained a Cronbach's alpha of 0.97 . This underlines the robust internal consistency of the inventory contents. This revised version of the instrument allows for gaining knowledge about the processes involved and applied in the formulation of intervention contexts, and gives guidance on monitoring, training and formation for the practice of Social Casework.

Keywords: inventory, social casework, intervention context, helping relationship, psychometric properties.

Recibido: 10/04/2016

Aceptado: 07/10/2016

Publicado: 20/12/2016

1.Departamento de Filosofía y Trabajo Social, Universidad de las Islas Baleares, España. E-mail: jp.cardona@uib.es

2. Departmento de Psicología, Universidad de las Islas Baleares, España. E-mail: mgili@uib.es

3. Universidad de las Islas Baleares, Departamento de Filosofía y Trabajo Social. Ctra. de Valldemossa, s/n. Ed. Beatriu de Pinós, D3, 07122 Palma de Mallorca, España. E-mail: quico.campos@uib.es 


\section{Extended Abstract}

Introduction. The term context is used by Watzlawick, Weakland and Fish (1976) to describe various situations of interaction in which each person takes on different conducts according to the circumstances in which they are interacting. Bateson (1977) refers to context when he points out that this is the relational framework in which the participants' roles and their relational messages become meaningful. In Social Work practice, the definition of the intervention context for change involves sharing together with the client (an individual or family) the definition of the problem and the possible solutions, agreeing on the objective and expectations on the help process, as well as the conditions in which such process will develop. All these aspects have to be known, shared and accepted by the parties in the help system in order to create a collaborative relationship within the service framework.

When we refer to professional intervention contexts we do not mean broad social contexts or environmental features. We are referring to the different relational agreements that take place within the framework of a helping relationship with individuals or families: the subjects to be dealt with and how to conduct the interventions. The definition of the intervention context is the result of mutually agreed working arrangements, which are explicit and acknowledge by all the parties in the help system. Such system, formed by professional and client (individual or family), will work cooperatively for a particular period of time to pursue the objectives agreed by the system itself in a shared and accepted process.

This perspective, already described by Carpenter (1993) and later developed by authors following the Strategic Solution Focused Approach (De Shazer,1988; O'Hanlon and Werner-Davis, 1990, O`Hanlon, 2001; Selekman, 1996 and Lipchik, 2004), breaks with the old approach in which the professional is the one to tell what to do and how to do it: with different responsibilities, it places us again in collaborative relationships of a symmetrical nature.

In professional practice of Social Casework (with individuals or families) eight intervention contexts have been clearly identified: assistance, informative, counselling, clinical, monitoring, assessment, training and mediation. In our formulation, the construction of a professional intervention context is closely linked to practices that are focused on generating a help alliance in its engagement dimension (Escudero, 2009), and it formalizes in a conjoint agreement on the objectives, goals and intervention method. The relational foundation supporting the construction of the intervention context is based on the widening of competences by means of cooperation practices (O'Hanlon, 2001), the generation of a therapeutic alliance (Friedlander, Escudero y Heatherington, 2009), and in conducting the intervention within a deontological and professional framework (Hamilton,1960) by means of practices based on a mutual conception of the helping relationship (White, 2002) and practices that are based on non-violent communication (Rosemberg, 2006).

In the inventory below, the definition of the intervention context is built together with the client in a process that goes through different stages linked to the Social Casework procedure, understood cooperatively (Cardona, 2012) and which can be tracked down at three times: a first moment connected to the initial interview or first encounters known as preliminary context, a second stage of study-comprehension interviews known as co-evolution of intervention context, and a third stage in the co-diagnostic interview known as intervention context for change. The definition of the intervention context is constantly adjusted and jointly constructed during the whole process and at every meeting with the client.

The definition of a preliminary intervention context is built in the initial interview or during the first encounters with the client. It is linked to the joint analysis, made by professional and client, of the request and it formalizes in a preliminary agreement based on this analysis. Client and Social Worker agree to start a help process with the initial aim of changing or improving a particular situation. For this purpose, an initial commitment to study and understand the situation will be established. It is a flexible commitment that will allow the modification or widening of the initial aims of the helping relationship. Clients should feel that their collaboration in the setting up of initial objectives is valued and their opinions about the help process are taken into account.

As the establishment of the alliance proceeds, the preliminary intervention context originally agreed usually coevolves so that it adjusts to the family's real needs. The family frequently feels more confident and open to share and ask for help for other needs or problems of a different kind to the ones that were initially raised. In these cases, if the help system assesses the possibility of expanding the initial agreement with other contents, the initially agreed context may coevolve into an intervention context which is different from the original one, or it may be broadened into two different intervention contexts at the same time. In the latter case, it may start a helping relationship from an assistance context, linked to a benefit, and coevolve towards an intervention context connected to counselling.

The definition of the intervention context for change is built at the co-diagnostic interview, once the study interviews have concluded. This interview enables the sharing and adjustment of diagnostic impressions from the study-comprehension process and, once the objectives and goals for change have been determined, it is possible to establish (together with the individual or family) the elements that define the professional intervention context for change. This 
is about confirming that the subjects to improve or change (objectives and goals for change) are clear for both, and also, about sharing with the individual or family the procedure which will be used in the interviews for change. Certain aspects must be made clear: the characteristics that the interviews and encounters of intervention for change, the nature of the relationship, the locations and times, the service requirements in order to start that process of intervention, confidentiality, intensity, limits and scope of the intervention that will be carried out by mutual agreement. According to the nature of the problem and objectives for change that have been agreed, the intervention context will involve a different kind of relationship and a distinctive intervention method.

The instruments that we put forward below derive from an orderly, congruent, and shared process that transversally gathers up intervention criteria focused on the generation of a therapeutic alliance and the cooperation that lies in the helping relationship from the initial contacts to the assessment stage (co-diagnostic).

This research aims at analysing the psychometric features in the Inventory for the analysis of the relation of help between the Social Worker and the Client during the phase of study and evaluation of the analysis of the problem situation (Cardona y Campos, 2009). This instrument is designed in order to find out the intervention criteria that social workers typically put into practice so that, together with their clients, they can determine what the intervention context for change will be. The instrument is useful in order to assess the way in which professionals guarantee efficient practice to explore and reinforce the family/client system and an adequate formulation of the intervention context within an explicit and joint agreement between the professional and the client. The theoretical foundations for this inventory are not based on a single model. They are related to the social casework tradition (Hamilton,1940; Hollis,1949; Aponte, 1976; Perlman, 1980; Rosell, 1989 and Azpeitia et al, 2003), the development of particular contexts (Ituarte, 1992; Cirillo, 1994; Ripol-Millet, 2001), and the latest trends (problem-focused approach, solution-focused approach, and narrative approach) linked to the systemic approach tradition (Satir, 1986; de Shazer;1988 ; Carpenter,1993; White, 1993, 2002, 2007; Selekman, 1996; Imbre-Black, 2000; O`Hanlon, 2001; Lipchik, 2004; Beyebach, 2006 and Friedlander, Escudero, y Heatherington, 2009).

Objective: This paper aims at analysing psychometric properties in the Inventory for the analysis of the relation of help between the Social Worker and the Client during the phase of study and evaluation of the analysis of the problem situation (Cardona and Campos, 2009). This instrument is designed in order to find out the intervention criteria that social workers typically put into practice so that, together with their clients, they can determine what the intervention context for change will be. This research refines the instrument by paying particular attention to the internal consistency criteria and provides a second version of such instrument.

Material and methods: the intentional non-probabilistic sample consists of the total number of social workers who work on projects involving direct contact with the individual or family. A total number of 115 individuals took part, of which $9.5 \%$ were men and $90.5 \%$ were women. A number of 84 (73\%) professionals answered the questionnaire, whilst 31 professionals refused to take part or were unable to do it.

The initial instrument, made up of 137 ítems, aimed at identifying and assessing the intervention criteria which were most frequently applied when determining the intervention context. The instrument was revised by experts in order to determine its logical validity and its contents. The KaiserMeyer-Olkin index (K.M.O.=0.88) and Bartlett's test of sphericity $(\mathrm{p}<0.05)$ indicated the statistical possibility of carrying out a factor analysis of the inventory. After that, the instrument was refined by performing an exploratory analysis of its principal components and a factor analysis with varimax rotation, and the reliability coefficient was determined (Cronbach's alpha).

Results and conclusions: the list of 137 ítems was reduced to 75 . The final factorial solution showed that the questionnaire was formed by a single dimension, along with the process logic of the instrument. The coefficient of internal consistency obtained a Cronbach's alpha of 0.97 . This underlines the robust internal consistency of the inventory contents. This revised version of the instrument allows for gaining knowledge about the processes involved and applied in the formulation of intervention contexts, and gives guidance on monitoring, training and formation for the practice of Social Casework. The instrument can guide professional practice in primary and specialized care services. It maintains an internal logic in the procedure due to the fact that, being one-dimensional, it is ordered and guided by a formulation of the intervention process in Social Casework, understood in a cooperative way. This enables the instrument to be easily formalised, reconverting its function as identification and analysis instrument into an intervention protocol. Finally, it should be pointed out that the usefulness of the instrument also lies in its potential use in monitoring processes and case analysis, as it provides specific, diverse and transversal intervention criteria, which the monitor and monitored can verify and analyse their practice.

Key words: inventory, social casework, intervention context, helping relationship, psychometric properties. 


\section{Introducción. La definición del contexto de intervención}

El término contexto es utilizado por Watzlawick, Weakland y Fish (1976) para describir las diversas situaciones de interacción en las cuales cada persona asume conductas distintas según el medio en que interactúa. Bateson (1977) hace referencia al contexto cuando señala que éste es el marco relacional en el cual las conductas de los partícipes y sus mensajes relacionales se hacen significativos. Desde nuestro enfoque, en la práctica del Trabajo Social, definir el contexto de intervención para el cambio implica compartir con el cliente (una persona o una familia) la definición del problema y las soluciones posibles, acordar la finalidad y las expectativas del proceso de ayuda, así como las condiciones desde las cuales se desarrollará dicho proceso. Estos aspectos deben ser conocidos, compartidos y aceptados por todas las personas que forman el sistema de ayuda al objeto de crear una relación colaborativa en el marco de un servicio.

Cuando nos referimos a contextos de intervención profesional, no nos referimos ni contextos sociales amplios ni a características del entorno. Nos referimos a los diferentes acuerdos relacionales que se dan en el marco de una relación de ayuda con personas o familias: cuáles serán los asuntos a tratar y cómo se van a llevar a cabo las intervenciones. Así pues, cada contexto puede entenderse como una plataforma desde la cual el tipo de relación terapéutica que se establece, mantiene una coherencia con el problema presentado y el método acordado para abordar dicha situación. Dicha plataforma está inmersa en un sistema de relaciones vinculadas a la cultura y al territorio, alejada de construcciones narrativas individualistas y dominantes y sostenidas sobre ideas y prácticas éticas, narrativas y de cooperación.

Definir el contexto de intervención profesional es el resultado de un acuerdo conjunto de trabajo, explícito y conocido por todos los componentes del sistema de ayuda. Este, constituido por profesional y cliente (persona o familia), trabajará cooperativamente durante un determinado período de tiempo con la intención de alcanzar los objetivos acordados por el propio sistema, mediante un procedimiento conocido y aceptado. Es el resultado de la voluntad de los actores (profesional y cliente) los cuales deciden, de forma colaborativa, unir esfuerzos para alcanzar metas conocidas (Cardona y Campos, 2009). Esta perspectiva, ya descrita en su momento por Carpenter (1993) y posteriormente desarrollada por todos los autores del Modelo Estratégico Centrado en Soluciones (De Shazer,1988; O`Hanlon y Werner-Davis, 1990, O`Hanlon, 2001; Selekman, 1996 y Lipchik, 2004), rompe la vieja perspectiva por la cual es el profesional el que dice qué hay que hacer y de qué manera: 
con responsabilidades distintas, nos reubica en relaciones colaborativas de naturaleza simétrica.

En la práctica profesional del Trabajo Social con casos se han identificado ocho contextos de intervención claramente diferenciados: asistencial, informativo, asesoramiento, clínico, control, evaluación, formación y mediación. Desde nuestro punto de vista, Hamilton (1960) ya formula por primera vez la necesidad de definir el contexto de intervención durante los primeros contactos entre profesional y cliente. Sin embargo, no será hasta las primeras investigaciones de D’Adda y Gallione (1983) donde se constata que las peticiones de ayuda de los clientes de los servicios sociales tienden a desarrollar cuatro tipos de contextos: informativo, asesoramiento, asistencial y control. Campanini y Luppi (1991) recogiendo la aportación anterior, realizan un amplio análisis aportando claves de comprensión relacionales de cada contexto, incorporando el contexto evaluativo a los ya descritos. Lamas (1997) plantea que los primeros encuentros entre profesional y cliente están enmarcados en diferentes contextos que se pueden dividir en seis categorías: asistencial, de consulta, terapéutico, de evaluación, de control e informativo. Por último Ripol-Millet (2001) añade a la ya mencionada clasificación un nuevo contexto, el contexto de mediación.

No cabe duda que la formulación del contexto de intervención no es un hecho aislado y azaroso, sino más bien al contrario. En el inicio de la relación de ayuda el profesional, conscientemente, pone en marcha intervenciones dirigidas a generar una alianza de ayuda en la dimensión de enganche: el cliente (persona o familia) puede percibir que su colaboración en las metas y los objetivos es valorada, que sus opiniones sobre la intervención son tenidas en cuenta, que sus puntos fuertes son potenciados y que los pequeños cambios que se van logrando son amplificados. En la formulación que presentamos, la construcción del contexto de intervención profesional está íntimamente relacionada con prácticas centradas en generar una alianza de ayuda en su dimensión enganche (Escudero, 2009), y se concreta en un acuerdo conjunto sobre los objetivos, las metas y el método de intervención. La base relacional que sustenta la construcción del contexto de intervención, a nuestro entender, se apoya en la ampliación de competencias a través de prácticas de cooperación (O`Hanlon, 2001), en la generación de una alianza terapeútica (Escudero, 2009), y en conducir la intervención dentro del marco de la deontología y ética profesional (Hamilton,1960) a través de prácticas basadas en una concepción recíproca de la relación de ayuda (White, 2002) y prácticas basadas en la comunicación no violenta (Rosemberg, 2006).

En el inventario que se presenta, la definición del contexto de intervención con el cliente se va construyendo a través de un proceso que transcurre por 
diferentes momentos ligados al procedimiento del Trabajo Social de Casos, entendido éste de forma cooperativa (Cardona, 2012) y que se concreta en tres momentos: un primer momento ligado a la entrevista de primer contacto o primeros encuentros denominado contexto preliminar, un segundo momento ubicado en el transcurso de las entrevistas de estudio-comprensión denominado co-evolución del contexto de intervención y un tercer momento ubicado en la entrevista de co-diagnóstico denominado contexto de intervención para el cambio. En el transitar de un momento a otro, la definición del contexto de intervención se va ajustando y co-construyendo en cada encuentro con el cliente.

La definición del contexto de intervención preliminar se construye en la entrevista de primer contacto o durante los primeros encuentros con el cliente. Está ligada al análisis de la demanda que, de forma compartida, se realiza con el cliente y se concreta en un acuerdo preliminar derivado de dicho análisis. Cliente y Trabajador Social acuerdan iniciar un proceso de ayuda con el objetivo inicial de cambiar o mejorar una situación determinada. Para ello, se establecerá un compromiso inicial destinado a estudiar y comprender dicha situación. Se trata de un compromiso flexible que va a permitir modificar o ampliar los objetivos iniciales de la relación de ayuda. El cliente debe percibir que su colaboración en los objetivos iniciales es valorada y que sus opiniones sobre el proceso de ayuda son tenidas en cuenta.

En la medida que se avanza en el establecimiento de la alianza, el contexto de intervención preliminar inicialmente acordado, suele co-evolucionar ajustándose más a las necesidades reales de la familia. Es frecuente que la familia pueda sentirse más confiada a compartir y solicitar ayuda para otras necesidades o problemas de naturaleza distinta a los planteados inicialmente. En estos casos, si el sistema de ayuda valora la posibilidad de ampliar el acuerdo inicial con otros contenidos, el contexto inicialmente acordado puede co-evolucionar dando lugar a un contexto de intervención diferente al inicial o bien ampliarse a dos contextos de intervención diferentes a la vez. En este último supuesto, se puede dar el caso de iniciar una relación de ayuda desde un contexto asistencial, ligado a una prestación y co-evolucionar hacia un contexto de intervención ligado a intervenciones de asesoramiento.

Una vez finalizadas las entrevistas de estudio, la definición del contexto de intervención para el cambio se construye durante la entrevista de co-diagnóstico. Ésta entrevista permite compartir y ajustar las impresiones diagnósticas extraídas del proceso de estudio-comprensión y, una vez definidos los objetivos y las metas de cambio, concretar con la persona o la familia, los elementos que definen el contexto de intervención profesional para el cambio. Se trata de confirmar que 
los temas a mejorar o cambiar son claros para ambos (objetivos y metas de cambio), así como el compartir con la persona o familia el procedimiento desde el cual se iniciarán las intervenciones para el cambio. Deben quedar claras las características que tendrán las entrevistas y encuentros de intervención para el cambio, la naturaleza de nuestra relación, los espacios y los tiempos, los requisitos del servicio para iniciar dicho proceso de intervención, la confidencialidad, la intensidad, los límites y el calado de la intervención que juntos y de mutuo acuerdo se van a llevar a cabo. Según la naturaleza del problema y los objetivos de cambio acordados, el tipo de contexto de intervención para el cambio desde el cual se encauzará la relación de ayuda, comportará una naturaleza de relación diferente y un método de intervención diferenciado.

Los contextos de intervención para el cambio en trabajo social tienen nombre. La dirección del cambio queda definida por los objetivos, las metas y los caminos a transitar para lograrlo tienen identidades metodológicas diferenciadas. Se pueden identificar métodos de intervención para cada contexto de intervención para el cambio. Esta realidad permite alejarse de lo genérico e investigar las prácticas y la intervención, al mismo tiempo que genera más identidad profesional.

El instrumento que presentamos resultado de un proceso ordenado, congruente y compartido, que recoge de forma transversal criterios de intervención centrados en la generación de una alianza terapéutica y en la cooperación que transita por la relación de ayuda desde los primeros contactos hasta el momento de evaluación (codiagnóstico).

Este trabajo tiene como objetivo analizar las características psicométricas del Inventario para el análisis de la relación de ayuda entre el Trabajador Social y el Cliente durante la fase de estudio y evaluación de la situación problema (Cardona y Campos, 2009). El instrumento está diseñado para conocer cuáles son los criterios de intervención que los trabajadores sociales ponen preferentemente en práctica para determinar, junto a sus clientes, cual será el contexto de intervención para el cambio. El instrumento permite calibrar de qué manera los profesionales aseguran una práctica eficaz para explorar y potenciar el sistema familiar/cliente y una adecuada formulación del contexto de intervención en un acuerdo explícito entre profesional y cliente. Así mismo, el trabajo pretende depurar el instrumento en base a sus características de consistencia interna y poder disponer de una segunda versión reducida del mismo. En el presente trabajo no se contemplan todos los procedimientos propios de la validación de un cuestionario sino que, una vez extraídos los ítmes que recojen de manera más adecuada la relación de ayuda en el Trabajo Social de Casos, se presentará una segunda versión del inventario que deberá ser validada con posterioridad. 
La estructura interna del inventario se apoya en una formulación teóricapráctica del Trabajo Social con Casos (Cardona, 2012). Está sustentada en bases relacionales sobre el establecimiento de la cooperación y la alianza de ayuda, como facilitadores para la comprensión de la situación problema y la construcción del contexto de intervención profesional con el cliente. A la hora de estructurar los contenidos, hemos tenido en cuenta que existe un acuerdo unánime en otorgar gran relevancia a las intervenciones encaminadas a construir una relación vincular, siendo estas consideradas una parte estructural del proceso. De forma transversal, el inventario presenta criterios de intervención referidos al establecimiento de relaciones basadas en la cooperación (O’Hanlon, 2001), la construcción de una alianza terapéutica (Escudero y López, 2003; Friedlander, Escudero y Heatherington, 2009), el establecimiento de relaciones reciprocas (White, 2002), deontología y ética profesional (Anderson, 1999; Hamilton, 1960 y Tuerlinck, 1973) y la detección de pautas cooperativas en los clientes para posteriormente poder cooperar con ellos en su mismo estilo (De Shazer, 1992; Selekman, 1996). Criterios de intervención referidos al joining como actitud colaborativa, se despliegan a lo largo del inventario, en diferentes prácticas como destacar las competencias y fortalezas familiares y transmitir verdadero afecto hacia los miembros, entre otros, facilitando así la generación de un clima emocional en el estudio con la familia (Minuchin,1974; Minuchin y Fishman,1981) además de facilitar al cliente entrar en contacto consigo mismo despertando y resaltando sus valores y sus competencias (Satir, 1986).

Las bases teóricas del inventario no se centran en un único modelo. Están vinculadas a la tradición del trabajo social de casos (Hamilton,1940; Hollis,1949; Aponte, 1976; Perlman, 1980; Rosell, 1989 y Azpeitia et al, 2003), al desarrollo de contextos específicos (Ituarte, 1992; Cirillo, 1994; Ripol-Millet, 2001), y a las últimas vanguardias (modelo centrado en problemas, modelo centrado en la solución y el modelo narrativo) ligadas a la tradición de la perspectiva sistémica (Satir, 1986; de Shazer;1988 ; Carpenter,1993; White, 1993, 2002, 2007; Selekman, 1996; Imbre-Black, 2000; O’Hanlon, 2001; Lipchik, 2004; Beyebach, 2006 y Friedlander, Escudero, y Heatherington, 2009).

En general, los criterios de intervención del inventario se desarrollan a través de un diálogo relacional entre profesional y cliente, donde el concepto de recoger datos propio de un observador de la primera cibernética, queda desplazado en pro de co-construir informaciones con el cliente, propio de un sistema que se transforma a través de su interacción (Andolfi, 2003): de los sistemas observados (primera cibernética) a los sistemas observantes (segunda cibernética). 
Tras lo anteriormente comentado, el objetivo de nuestro estudio es analizar las características psicométricas del Inventario para el análisis de la relación de ayuda entre el Trabajador Social y el Cliente durante la fase de estudio y evaluación de la situación problema (Cardona, 2008) Así mismo, el trabajo pretende depurar el instrumento en base a sus características de consistencia interna y poder disponer de una segunda versión reducida del mismo.

\section{Metodología}

1.Muestra . La muestra, no probabilística e intencional, estuvo compuesta por el total de trabajadores sociales ubicados en proyectos de atención directa individual y familiar. Participaron un total de 115 sujetos, de los cuales 9,5\% eran hombres y el 90,5\% mujeres. Respondieron al cuestionario 84 profesionales $(73,04 \%)$ y 31 personas rechazaron la invitación a participar o no pudieron hacerlo. No trabajar directamente en proyectos de intervención fue considerado un motivo de exclusión. Los años de experiencia profesional de los profesionales de la muestra se concentraron especialmente en tres intérvalos: de entre 0 y 5 años, con un 24\%; entre 6 y 10 años, con un 28,8\% y entre 16 y 20 años con un $26,4 \%$. La media de años de experiencia profesional fue $15(\mathrm{Sd}=7.3)$.

2. Construcción del instrumento. Se revisó la literatura sobre metodología para la construcción y validación de cuestionarios (Feinstein, 1987), los conceptos relacionados con la definición del contexto de intervención en el marco de la relación de ayuda, los dominios que la integran y su asociación con la evaluación de la situación problema por parte de los trabajadores sociales. La revisión sistemática efectuada en diversas bases de datos (Dialnet, Scopus, EbscoHostFamily Studies Abstracts, EbscoHost-Psycinfo, CINDOC, Social Work Abstracts), así como en las revistas de trabajo social del Journal Citation Reports (Research on Social Work Practice, Social Work, British Journal of Social Work, Journal of Social Work Practice, Clinical Social Work Journal y International Social Work), durante los períodos comprendidos entre 1990 y 2015, puso en evidencia que el concepto de contexto de intervención tiene una escasa presencia documental y no ha sido objeto de investigación. Se usaron los descriptores de búsqueda en inglés y castellano relacionados con el contenido del instrumento: contexto de intenverción, intervention context en lengua inglesa, en las modalidades A or B y A and B. Constatamos que no existían instrumentos de evaluación que permitieran conocer de qué manera y con qué criterios los profesionales y sus clientes adoptaban decisiones relacionadas con los acuerdos de trabajo que deben iniciarse en los procesos de cambio. Estas circunstancias motivaron nuestro interés en el diseño del instrumento. 
Finalizada la revisión, se elaboró la primera versión de un instrumento auto-administrado para evaluar la intervención en los primeros encuentros de la relación de ayuda en la práctica del trabajo social de casos, cuya finalidad es determinar cuáles son los procedimientos, habilidades y técnicas que los profesionales ponen en práctica en su intervención con casos.

El cuestionario estaba integrado inicialmente por 137 ítems, definidos como criterios de intervención ${ }^{1}$, que reflejan las descripciones de habilidades, técnicas, actitudes o estrategias presentes en las fases de estudio y evaluación en una relación de ayuda. Los 137 criterios de intervención se organizaron inicialmente en siete áreas. De ellas, cuatro se corresponden con los primeros momentos del método de investigación en Trabajo Social de Casos: acogida inicial, análisis de la demanda, estudio de la situación problema y por último (fruto de la evaluación diagnóstica compartida con el cliente), la definición de objetivos y metas concretados en un acuerdo mutuo de trabajo. Las tres áreas restantes además de ocupar un área específica en la organización del instrumento, se encuentran de forma transversal en todo el inventario, ya que de hecho, transitan durante todo el proceso de ayuda: la construcción de una relación de ayuda basada en la cooperación y la alianza de ayuda, ética y deontología y por último la construcción del contexto de intervención profesional. Para cada uno de los 137 criterios de intervención, se generaron dos ítems: Nivel de importancia y Nivel de realización, presentados con cinco alternativas de respuesta establecidos según una escala Likert en un formato numérico que van desde 1 (Ninguno) hasta 5 (Mucho) para el nivel de importancia y 1 (Nunca) hasta 5 (Siempre) para el nivel de realización. En el presente trabajo se utilizan los datos relativos a la escala Nivel de Importancia.

La validez lógica y de contenido se evaluó mediante consenso de expertos a través de dos profesores de dos universidades, especialistas en la materia. En esta primera versión también se aplicó el instrumento a un grupo piloto de cinco profesionales en activo de generaciones diferentes para evaluar el nivel de comprensión, legibilidad y reproducibilidad. Con posterioridad se volvió a aplicar a dos integrantes del grupo de expertos con el objetivo de eliminar aquellos ítems en que ambos estaban de acuerdo que introducían más ruido que claridad. Concluida la revisión del instrumento por el panel de expertos y efectuada la prueba piloto, se hicieron modificaciones en la redacción de algunos ítems, se eliminaron cinco y se ajustó la redacción en dos. El instrumento

1. . Término adoptado del National Occupational Standards for Social Work (May 2002), Leeds, TOPSS England 2004. Se entiende como criterio de intervención a aquella acción expresada en forma de habilidad, estrategia o técnica. 
final para su aplicación a la muestra de sujetos contenía 137 ítems agrupados en las siete áreas nombradas anteriormente (Cardona y Campos, 2009).

3. Administración del instrumento. Los participantes respondieron el cuestionario de manera auto-administrada en sus lugares de trabajo previa cita concertada. En el servicio se informó a los participantes de las características de la investigación y de las condiciones de cumplimentación del instrumento: procedimiento para responder, duración (una hora) y descanso (quince minutos), solicitándose durante el mismo el no hablar de los contenidos del inventario. Además se administró a los sujetos un cuestionario estructurado en siete bloques de preguntas: referidas a la ubicación del centro, organización, dotación e infraestructuras del servicio, la formación de base, y su actualización; experiencia y desarrollo profesional y apoyos exteriores. La investigación se ajustó a las directrices éticas relevantes para los estudios con personas, y a las normas éticas aceptadas internacionalmente por las directrices profesionales pertinentes.

4. Análisis de los datos. En primer lugar y con el objetivo de eliminar ambiguedades, redundancias e incoherencias, se realizó una depuración del instrumento, eliminando los criterios de intervención que se ha demostrado que no son útiles a la vista del análisis estadístico, mejorando el estilo y redacción de los restantes. Posteriormente se analizaron sus propiedades psicométricas y finalmente se realizó un análisis descriptivo de los ítems. Se realizó un análisis exploratorio de componentes principales y posteriormente un análisis factorial con rotación varimax. La consistencia interna del cuestionario se determinó mediante el coeficiente de fiabilidad (alfa de Cronbach). En el análisis de datos se utilizó el paquete estadístico SPSS 21 para Windows.

\section{Resultados}

Una vez aplicado el instrumento, se depuraron los ítems aplicando un criterio relativo a la importancia de las intervenciones. Se eliminaron del cuestionario todos aquellos ítems en los que la suma de frecuencias "Mucho" y "Bastante" no fue considerada al menos por el $90 \%$ de la muestra. Los ítems cuya correlación entre su calificación con el total de su dominio y con el total del cuestionario fue menor a 0.20 también fueron eliminados por su escasa aportación a la calificación total. Tras esta depuración inicial el banco de ciento trenta y siete (137) ítems quedó reducido a ochenta y tres (83).

De manera previa a la realización del análisis factorial, se analizó la adecuación muestral de los ítems. El índice Kaiser-Meyer-Olkin (K.M.O.=0.88) y la prueba de esfericidad de Bartlett $(\mathrm{p}<0.05)$ indicaron la posibilidad estadística 
de realizar el análisis factorial del inventario. A continuación se procedió a un primer análisis exploratorio de componentes principales, utilizando los autovalores mayores a uno y seleccionando aquellos ítems que saturaran en los factores seleccionados con una carga factorial de al menos 0.40. La Tabla I refleja el índice de saturación de cada uno de los 75 criterios de intervención que constituyen el instrumento.

Tabla I.

Análisis factorial exploratorio: índice de saturación de cada ítem

\begin{tabular}{|l|c|c|c|c|c|c|c|c|c|c|c|c|c|c|c|}
\hline Ítem & 1 & 2 & 3 & 4 & 5 & 6 & 7 & 8 & 9 & 10 & 11 & 12 & 13 & 14 & 15 \\
\hline Satur & .510 & .403 & .549 & .656 & .412 & .435 & .471 & .497 & .455 & .656 & .460 & .598 & .477 & .458 & .440 \\
\hline Ítem & 16 & 17 & 18 & 19 & 20 & 21 & 22 & 23 & 24 & 25 & 26 & 27 & 28 & 29 & 30 \\
\hline Sat. & .618 & .697 & .426 & .487 & .593 & .446 & .482 & .516 & .414 & .617 & .445 & .567 & .435 & .567 & .543 \\
\hline Ítem & 31 & 32 & 33 & 34 & 35 & 36 & 37 & 38 & 39 & 40 & 41 & 42 & 43 & 44 & 45 \\
\hline Sat. & .696 & .592 & .674 & .543 & .579 & .451 & .546 & .446 & .607 & .498 & .495 & .457 & .528 & .436 & .436 \\
\hline 36 & 46 & 47 & 48 & 49 & 50 & 51 & 52 & 53 & 54 & 55 & 56 & 57 & 58 & 59 & 60 \\
\hline Sat. & .476 & .577 & .606 & .550 & .562 & .448 & .438 & .445 & .561 & .507 & .683 & .639 & .551 & .430 & .501 \\
\hline Ítem & 61 & 62 & 63 & 64 & 65 & 66 & 67 & 68 & 69 & 70 & 71 & 72 & 73 & 74 & 75 \\
\hline Sat. & .419 & .602 & .662 & .595 & .584 & .747 & .598 & .542 & .629 & .618 & .598 & .491 & .516 & .492 & .403 \\
\hline
\end{tabular}

* Índice de saturación

Los datos obtenidos tras el análisis exploratorio incrementó ligeramente el número de ítems eliminados (ocho) hasta quedar en un total final de setenta y cinco. La comparación entre el primer instrumento y el instrumento final muestra una diferencia de sesenta y dos ítems (62). La inexistencia de instrumentos similares hace imposible la comparación y la evaluación de la validez de criterio concurrente.

Una vez efectuada una primera depuración del instrumento inicial, el grupo de expertos académicos facilitó el ajuste de los niveles de comprensión, legibilidad y reproducibilidad. Su contribución se orientó especialmente a señalar la necesidad de una mayor clarificación en la redacción de diversos ítems del instrumento. El grupo de expertos profesionales valoró el tiempo de aplicación requerido, la adecuación al perfil del profesional al que se dirigía (criterio de necesidad de entrenamiento) y el formato inicial propuesto. La validez aparente quedó asegurada con la participación de los dos grupos y su validación mediante juicios. Las pruebas preliminares efectuadas aseguraron los parámetros de homogeneidad al cumplir los expertos características sociodemográficas similares. 
Propiedades psicométricas del Inventario para la construcción del contexto de...

Tabla II.

Estadísticos descriptivos

\begin{tabular}{|c|c|c|c|c|c|c|}
\hline $\begin{array}{c}\text { Ítem } \\
\text { número }\end{array}$ & $\mathrm{N}$ & Mínimo & Máximo & Media & $\begin{array}{c}\text { Desviación } \\
\text { estándar }\end{array}$ & Varianza \\
\hline 1 & 84 & 3 & 5 & 4,75 & 0,512 & 0,262 \\
\hline 2 & 84 & 4 & 5 & 4,73 & 0,449 & 0,201 \\
\hline 3 & 84 & 1 & 5 & 4,33 & 0,766 & 0,586 \\
\hline 4 & 82 & 2 & 5 & 4,56 & 0,687 & 0,472 \\
\hline 5 & 83 & 4 & 5 & 4,84 & 0,366 & 0,134 \\
\hline 6 & 84 & 4 & 5 & 4,81 & 0,395 & 0,156 \\
\hline 7 & 83 & 3 & 5 & 4,82 & 0,417 & 0,174 \\
\hline 8 & 83 & 3 & 5 & 4,66 & 0,547 & 0,299 \\
\hline 9 & 84 & 3 & 5 & 4,8 & 0,433 & 0,187 \\
\hline 10 & 82 & 3 & 5 & 4,72 & 0,504 & 0,254 \\
\hline 11 & 84 & 2 & 5 & 4,36 & 0,705 & 0,497 \\
\hline 12 & 82 & 2 & 5 & 4,54 & 0,773 & 0,597 \\
\hline 13 & 83 & 2 & 5 & 4,63 & 0,676 & 0,456 \\
\hline 14 & 84 & 3 & 5 & 4,4 & 0,661 & 0,437 \\
\hline 15 & 84 & 3 & 5 & 4,68 & 0,519 & 0,269 \\
\hline 16 & 83 & 1 & 5 & 4,24 & 0,82 & 0,673 \\
\hline 17 & 82 & 1 & 5 & 4,48 & 0,741 & 0,549 \\
\hline 18 & 83 & 2 & 5 & 4,51 & 0,669 & 0,448 \\
\hline 19 & 84 & 2 & 5 & 4,57 & 0,682 & 0,465 \\
\hline 20 & 84 & 3 & 5 & 4,65 & 0,57 & 0,325 \\
\hline 21 & 84 & 3 & 5 & 4,6 & 0,583 & 0,34 \\
\hline 22 & 84 & 2 & 5 & 4,56 & 0,683 & 0,466 \\
\hline 23 & 84 & 3 & 5 & 4,67 & 0,545 & 0,297 \\
\hline 24 & 84 & 3 & 5 & 4,73 & 0,499 & 0,249 \\
\hline 25 & 83 & 2 & 5 & 4,6 & 0,604 & 0,364 \\
\hline 26 & 82 & 2 & 5 & 4,44 & 0,739 & 0,546 \\
\hline 27 & 83 & 3 & 5 & 4,55 & 0,649 & 0,421 \\
\hline 28 & 84 & 3 & 5 & 4,56 & 0,628 & 0,394 \\
\hline 29 & 84 & 3 & 5 & 4,71 & 0,528 & 0,279 \\
\hline 30 & 84 & 3 & 5 & 4,6 & 0,583 & 0,34 \\
\hline 31 & 82 & 3 & 5 & 4,57 & 0,589 & 0,346 \\
\hline 32 & 83 & 3 & 5 & 4,53 & 0,612 & 0,374 \\
\hline 33 & 83 & 2 & 5 & 4,61 & 0,659 & 0,435 \\
\hline 34 & 84 & 2 & 5 & 4,38 & 0,743 & 0,552 \\
\hline 35 & 84 & 3 & 5 & 4,6 & 0,583 & 0,34 \\
\hline 36 & 81 & 1 & 5 & 4,19 & 0,91 & 0,828 \\
\hline 37 & 83 & 3 & 5 & 4,48 & 0,592 & 0,35 \\
\hline & & & & & & \\
\hline 13 & & 5 & & & \\
\hline
\end{tabular}

Alternativas. Cuadernos de Trabajo Social, 23, 2016, pp. 91-117 - ISSN 1133-0473 DOI: 10.14198/ALTERN2016.23.05 


\begin{tabular}{|c|c|c|c|c|c|c|}
\hline $\begin{array}{c}\text { Ítem } \\
\text { número }\end{array}$ & $\mathrm{N}$ & Mínimo & Máximo & Media & $\begin{array}{c}\text { Desviación } \\
\text { estándar }\end{array}$ & Varianza \\
\hline 38 & 84 & 3 & 5 & 4,81 & 0,452 & 0,204 \\
\hline 39 & 83 & 3 & 5 & 4,61 & 0,621 & 0,386 \\
\hline 40 & 84 & 3 & 5 & 4,65 & 0,549 & 0,301 \\
\hline 41 & 84 & 3 & 5 & 4,55 & 0,666 & 0,443 \\
\hline 42 & 84 & 3 & 5 & 4,65 & 0,591 & 0,349 \\
\hline 43 & 81 & 2 & 5 & 4,4 & 0,753 & 0,567 \\
\hline 44 & 82 & 3 & 5 & 4,7 & 0,581 & 0,338 \\
\hline 45 & 83 & 3 & 5 & 4,61 & 0,581 & 0,337 \\
\hline 46 & 84 & 3 & 5 & 4,68 & 0,584 & 0,341 \\
\hline 47 & 83 & 3 & 5 & 4,52 & 0,632 & 0,399 \\
\hline 48 & 84 & 2 & 5 & 4,33 & 0,665 & 0,442 \\
\hline 49 & 83 & 2 & 5 & 4,45 & 0,72 & 0,518 \\
\hline 50 & 83 & 2 & 5 & 4,87 & 0,463 & 0,214 \\
\hline 51 & 84 & 3 & 5 & 4,87 & 0,433 & 0,187 \\
\hline 52 & 84 & 3 & 5 & 4,76 & 0,481 & 0,232 \\
\hline 53 & 84 & 3 & 5 & 4,8 & 0,433 & 0,187 \\
\hline 54 & 84 & 3 & 5 & 4,77 & 0,475 & 0,225 \\
\hline 55 & 84 & 3 & 5 & 4,81 & 0,424 & 0,18 \\
\hline 56 & 84 & 3 & 5 & 4,9 & 0,334 & 0,111 \\
\hline 57 & 82 & 3 & 5 & 4,59 & 0,587 & 0,344 \\
\hline 58 & 84 & 3 & 5 & 4,63 & 0,576 & 0,332 \\
\hline 59 & 83 & 3 & 5 & 4,59 & 0,625 & 0,391 \\
\hline 60 & 83 & 3 & 5 & 4,72 & 0,477 & 0,227 \\
\hline 61 & 83 & 2 & 5 & 4,57 & 0,588 & 0,346 \\
\hline 62 & 81 & 2 & 5 & 4,46 & 0,672 & 0,451 \\
\hline 63 & 83 & 3 & 5 & 4,54 & 0,668 & 0,446 \\
\hline 64 & 81 & 3 & 5 & 4,54 & 0,571 & 0,326 \\
\hline 65 & 83 & 3 & 5 & 4,55 & 0,667 & 0,445 \\
\hline 66 & 84 & 2 & 5 & 4,5 & 0,685 & 0,47 \\
\hline 67 & 83 & 3 & 5 & 4,71 & 0,553 & 0,306 \\
\hline 68 & 84 & 3 & 5 & 4,67 & 0,581 & 0,339 \\
\hline 69 & 84 & 3 & 5 & 4,65 & 0,503 & 0,253 \\
\hline 70 & 82 & 3 & 5 & 4,7 & 0,537 & 0,289 \\
\hline 71 & 84 & 2 & 5 & 4,58 & 0,68 & 0,463 \\
\hline 72 & 84 & 2 & 5 & 4,56 & 0,628 & 0,394 \\
\hline 73 & 84 & 2 & 5 & 4,4 & 0,713 & 0,509 \\
\hline 74 & 84 & 2 & 5 & 4,51 & 0,649 & 0,422 \\
\hline 75 & 84 & 2 & 5 & 4,58 & 0,68 & 0,463 \\
\hline
\end{tabular}


Los ítems seleccionados fueron sometidos a un nuevo análisis factorial utilizando los mismos criterios. La solución factorial final mostró que el cuestionario estaba constituido por una única dimensión que explicaba el 37,96\% de la varianza total. El coeficiente de consistencia interna del cuestionario reflejó un alfa de Cronbach de 0.97.

\section{Discusión y Conclusiones}

La inexistencia de instrumentos similares hace imposible la comparación. En consecuencia, únicamente podemos comentar la validez y la fiabilidad de los resultados. La ausencia de tradición cuantitativa relacionada con la evaluación de las intervenciones que los trabajadores sociales realizan, ha sido una de las motivaciones que han impulsado la creación del instrumento.

El análisis de las características psicométricas de una escala es un proceso que comprende varias etapas y que requiere un número elevado de sujetos. En este caso, el tamaño de la muestra es uno de los elementos de debilidad del proceso de validación, aunque ello no implica que su consistencia interna sea puesta en entredicho.

Vistos los resultados del análisis factorial exploratorio, su consistencia interna y la constatación de su unidimensionalidad, consideramos que el instrumento recoge adecuadamente las acciones, formuladas en términos de criterios de intervención, que un profesional del trabajo social debe desarrollar en los primeros encuentros de la relación de ayuda al objeto de determinar, junto a sus clientes, el contexto de intervención para el cambio. Siendo unidimensional, el inventario recoge criterios de intervención relacionados con la acogida, la escucha y la formulación de las demandas, la comprensión de la situación problemática, aspectos pragmáticos ligados a la deontología profesional, el desarrollo de alianza de ayuda, la formulación de objetivos y la definición del contexto.

En relación a la primera formulación del instrumento, muy preciso y detallado en su contenido inicial, se han eliminado sesenta y dos criterios de intervención. La eliminación de los criterios de intervención redundantes ha mejorado la estructura y la capacidad de evaluación del instrumento. El detalle, por áreas, queda de la siguiente forma:

1. Acogida inicial. Se han mantenido 2 (22\%) y se han eliminado 7 $(77,8 \%)$ de los 9 criterios de intervención que constituían esta primera Área de Acogida Inicial. Ello nos induce a considerar que los profesionales desarrollan poco la denominada "fase social" de las entrevistas: dirigirse a las personas por sus nombres, explicar brevemente la 
función del servicio, en que consiste su trabajo o manifestar el interés por conocer y escuchar sus peticiones o consultas.

2. Escucha y ubicación de la demanda. Se han mantenido 13 (52\%) y se han eliminado 12 (48\%) de los 25 criterios de intervención que constituían esta área. Los ítems eliminados están ligados a la información relacionada con el contexto relacional de los clientes: la relación entre el tipo de situación problema y el ciclo vital de la persona o familia y la información relativa al trabajo en red y la vinculación de los clientes en redes de atención profesional.

3. Comprensión de la situación problema. Se han mantenido 23 (46,9\%) y se han eliminado $26(53,1 \%)$ de los 49 criterios de intervención que constituían esta área. Conocer cómo se genera la situación problema, cómo se mantiene a través del tiempo, cuáles han sido las soluciones intentadas en la secuencia temporal, cuáles han sido las experiencias de éxito o fracaso en relación a la situación problema, qué les impide cambiar o en qué momento del ciclo vital se ubica la demanda, no quedan a nuestro juicio, suficientemente reflejados en el instrumento. Sorprende la eliminación de la práctica de entrevistas a domicilio y un conjunto de ítems vinculados a la comprensión del ecosistema familiar de los clientes.

4. Concretar objetivos y construir un acuerdo mutuo. Se han mantenido $10(55,5 \%)$ y se han eliminado $8(48,5 \%)$ de los 18 criterios de intervención que constituían esta área. Desaparecen los ítems que se relacionan con la atribución de significado sobre la naturaleza y tipo de objetivo acordado.

5. Deontología, principios y valores. Se han mantenido el $100 \%$ de los ítems.

6. Alianza de ayuda. Se han mantenido 9 (75\%) y se han eliminado 3 (25\%) de los 12 criterios de intervención que constituían esta área. Se han eliminado los criterios de intervención que ponen de manifiesto la conexión emocional entre profesionales y clientes.

7. Definir el Contexto de intervención. Se han mantenido $9(56,2 \%)$ y se han eliminado $7(43,7 \%)$ de los 16 criterios de intervención que constituían esta área, relacionados con ítems que reflejan el procedimientos específico y detallado para poder definir el contexto.

En conjunto, consideramos que la perspectiva histórica relacionada con la aparición de los problemas (dimensión temporal) y sus intentos de solución por parte del cliente ha quedado en una posición débil. 
A pesar de ello, el instrumento afianza la perspectiva por la cual, en la fase de estudio de las situaciones problema de los clientes del trabajo social, no solo se hace necesario el conocimiento de la naturaleza de dichos problemas, sino también el de potenciar las competencias de los sistemas clientes mediante el análisis de las soluciones intentadas y las capacidades de los mismos. A lo largo de todo el instrumento se manifiesta claramente la transversalidad de los criterios de intervención que se basan en la alianza de ayuda, la cooperación y la formulación del contexto de intervención.

La reducción de sesenta y dos ítems en relación al instrumento original, nos ha obligado a introducir algunas aclaraciones (que en el instrumento final aparecen en cursiva) para facilitar la compresión del contenido y de proceso. Al tratarse de un instrumento unidimensional, la división en siete áreas efectuada en el instrumento original (Cardona y Campos, 2009) no queda explícitamente reflejada. Con la intención de no perder la lógica procesual del primer instrumento, se han efectuado pequeños cambios en el orden de los ítems.

A nuestro juicio, la utilidad del instrumento es evidente. Más allá de su valor en la identificación de cómo se determina el contexto de intervención, el instrumento puede orientar la práctica profesional en los servicios de atención primaria y especializada. Mantiene una lógica interna procesual, ya que al ser unidimensional, está ordenado y guiado por una formulación del proceso de intervención en el Trabajo Social con casos, entendido de forma cooperativa (Cardona 2012). Ello permite que el instrumento pueda ser protocolizado con suma facilidad, reconvirtiendo su utilidad, de instrumento de identificación y análisis (Cardona-Cardona, Montaño-Moreno, y Campos-Vidal, 2016) a protocolo de intervención. Por último, señalar que la utilidad del instrumento también radica en su posible utilización en los procesos de supervisión y análisis de casos, en la medida que proporciona criterios de intervención concretos, diversos y transversales con los cuales supervisor y supervisado pueden verificar y analizar la práctica. A continuación presentamos el instrumento. 


\section{Cuestionario}

\section{CCIP - vII}

Construcción del contexto de intervención profesional

Inventario para la construcción del contexto de intervención en el Trabajo Social de Casos

Versión II

(C) Josefa Cardona, 2015

El inventario está compuesto por un conjunto de criterios de intervención (acción expresada en forma de habilidad, estrategia o técnica) y se valora a través de dos dimensiones: nivel de importancia y nivel de realización. Los criterios de intervención están ordenados de forma secuencial según el procedimiento metodológico aplicado al Trabajo Social de Casos.

Se puede valorar cada uno de los enunciados en una escala que va del 1 al 5 considerando los siguientes valores:

\begin{tabular}{|c|c|c|}
\hline & Nivel de Importancia & Nivel de Realización \\
\hline 1 & Ninguno & Nunca \\
\hline 2 & Poco & Esporádico \\
\hline 3 & Suficiente & Frecuente \\
\hline 4 & Bastante & Casi siempre \\
\hline 5 & Mucho & Siempre \\
\hline
\end{tabular}

Se solicita al profesional que cumplimente cada casilla con un círculo, asignándole uno de los valores predeterminados en los cuadros superiores. 


\title{
CCIP - vII \\ Construcción del contexto de intervención profesional
}

\author{
Inventario para la construcción del contexto de intervención \\ en el Trabajo Social de Casos \\ (C) Josefa Cardona.
}

Este inventario puede ser usado libremente, siempre y cuando se atribuya la autoría, se cite correctamente y no se realicen cambios.

\begin{tabular}{|c|c|c|c|}
\hline & Criterios de realización & Importancia & Realización \\
\hline 1 & $\begin{array}{l}\text { Presentación recíproca entre Trabajador Social y la Persona o } \\
\text { Familia }\end{array}$ & 1123345 & 12345 \\
\hline 2 & Mirar a los ojos y /o dar la mano y /o invitar a tomar asiento & 122345 & 12345 \\
\hline 3 & $\begin{array}{l}\text { Reconocer los esfuerzos que la persona o la familia ha tenido que } \\
\text { poner en marcha para solicitar ayuda }\end{array}$ & 123345 & 12345 \\
\hline 4 & $\begin{array}{l}\text { Cuidar de forma especial la manera de recibir a la persona o familia } \\
\text { en los primeros encuentros para facilitar la generación de un buen } \\
\text { vinculo personal }\end{array}$ & $1 \quad 23045$ & 12345 \\
\hline 5 & $\begin{array}{l}\text { Tomarse un tiempo para escuchar la demanda y evitar proponer } \\
\text { soluciones hasta que no se haya comprendido y ubicado el } \\
\text { verdadero sentido de la demanda }\end{array}$ & 1123345 & 12345 \\
\hline 6 & $\begin{array}{l}\text { Activar la disposición para prestar atención, interés y motivación } \\
\text { para recibir la demanda (escucha activa) }\end{array}$ & $1 \quad 23045$ & 12345 \\
\hline 7 & $\begin{array}{l}\text { Activar la disposición para comprender y aproximarse al significado } \\
\text { (sentimientos, emociones, valores) que tienen los hechos para la } \\
\text { persona o para los miembros de la familia (escucha activa) }\end{array}$ & $1 \quad 23045$ & 12345 \\
\hline 8 & $\begin{array}{l}\text { Con actitud receptiva, clarificar las palabras o contenidos que no } \\
\text { entendemos del relato que exponen las personas }\end{array}$ & 1123345 & 12345 \\
\hline 9 & $\begin{array}{l}\text { Generar confianza en la relación profesional para facilitar a la } \\
\text { persona o familia exponer sus problemas y necesidades que } \\
\text { subyacen en la demanda inicial }\end{array}$ & $1 \quad 23045$ & 12345 \\
\hline 10 & $\begin{array}{l}\text { Mostrar comprensión empática ante la situación expuesta y respeto } \\
\text { incondicional hacia la persona }\end{array}$ & 1123345 & 12345 \\
\hline 11 & $\begin{array}{l}\text { Conocer los valores propios de la persona o familia y los de su } \\
\text { grupo social de pertenencia para comprender mejor cómo vive la } \\
\text { situación y lo que significa en su vida }\end{array}$ & 1123345 & 12345 \\
\hline 12 & $\begin{array}{l}\text { Conocer los valores propios del Trabajador Social y mantenerlos } \\
\text { en quietud para comprender la situación desde el significado } \\
\text { que la persona o la familia le da a los hechos y no desde nuestros } \\
\text { significados (auto-conocimiento) }\end{array}$ & 1123345 & 12345 \\
\hline 13 & $\begin{array}{l}\text { En el caso de que la demanda la presente otra persona distinta al } \\
\text { cliente, conocer a la persona cliente o familia y saber por ella, de su } \\
\text { necesidad o problema }\end{array}$ & $1 \quad 2 \quad 3 \quad 45$ & 123345 \\
\hline 14 & $\begin{array}{l}\text { Demostrar interés en conocer a los miembros de la familia para } \\
\text { facilitar la comprensión de su sistema relacional }\end{array}$ & $\begin{array}{lllll}1 & 2 & 3 & 4 & 5\end{array}$ & 12345 \\
\hline 15 & $\begin{array}{l}\text { Entender en qué desean ser ayudados y cómo han pensado que se } \\
\text { concrete la ayuda }\end{array}$ & 123345 & 12345 \\
\hline
\end{tabular}

Alternativas. Cuadernos de Trabajo Social, 23, 2016, pp. 91-117 - ISSN 1133-0473

DOI: 10.14198/ALTERN2016.23.05 


\begin{tabular}{|c|c|c|c|}
\hline & Criterios de realización & Importancia & Realización \\
\hline 16 & $\begin{array}{l}\text { Conocer cuál es la propuesta de relación que la persona o familia } \\
\text { propone al trabajador social (Junto con la demanda, la persona } \\
\text { también solicita al trabajador social un trato, una forma de relación: } \\
\text { delegar la responsabilidad, pasividad, colaboración...) }\end{array}$ & 123345 & 12345 \\
\hline 17 & $\begin{array}{l}\text { Si es necesario, redefinir la propuesta de relación en una relación } \\
\text { colaborativa, que esté basada en su competencia, más que en sus } \\
\text { déficits }\end{array}$ & $\begin{array}{lllll}1 & 2 & 3 & 4 & 5\end{array}$ & 12345 \\
\hline 18 & $\begin{array}{l}\text { Ubicar a la persona en su sistema: visualizar a la persona en su } \\
\text { núcleo familiar y social de referencia }\end{array}$ & 122345 & 12345 \\
\hline 19 & $\begin{array}{l}\text { Elaborar con la persona y/o con la familia su genograma } \\
\text { y su ecomapa para conocer su composición familiar, las relaciones } \\
\text { entre sus miembros y su red de apoyo }\end{array}$ & 122345 & 12345 \\
\hline 20 & $\begin{array}{l}\text { Cuando la persona o familia comenta una situación positiva o de } \\
\text { mejora, dedicar un espacio de la conversación a elogiar y mostrar } \\
\text { satisfacción (joining) }\end{array}$ & 123345 & 12345 \\
\hline 21 & $\begin{array}{l}\text { Conocer el nivel de conciencia que tiene la persona o familia de sus } \\
\text { dificultades y limitaciones, de sus capacidades y apoyos para hacer } \\
\text { frente a la situación }\end{array}$ & $\begin{array}{lllll}1 & 2 & 3 & 4 & 5\end{array}$ & 12345 \\
\hline 22 & $\begin{array}{l}\text { Distinguir entre un funcionamiento patológico, uno disfun } \\
\text { una etapa crítica que la persona o familia pueda estar atrav }\end{array}$ & 122345 & 12345 \\
\hline 23 & $\begin{array}{l}\text { Identificar comportamientos y/o situa } \\
\text { protección que esté viviendo la perso }\end{array}$ & 123345 & 12345 \\
\hline 24 & $\begin{array}{l}\text { Conocer el grado de implicación y motivación que presenta la } \\
\text { persona o familia para hacer frente a la situación- problema }\end{array}$ & 122345 & 12345 \\
\hline 25 & $\begin{array}{l}\text { Ser consciente del grado de impl } \\
\text { el Trabajador Social para trabajar } \\
\text { situación problema (auto-conoci }\end{array}$ & 123345 & 12345 \\
\hline 26 & $\begin{array}{l}\text { nas profesionales y } \\
\text { s con el caso }\end{array}$ & $\begin{array}{lllll}1 & 2 & 3 & 4 & 5\end{array}$ & 12345 \\
\hline 27 & $\begin{array}{l}\text { uestro papel en la red asistencial que está } \\
\text { o }\end{array}$ & 123345 & 12345 \\
\hline 28 & $\begin{array}{l}\text { Construida una primera valoración de la situación problema- } \\
\text { necesidad, valorar con la persona o familia la necesidad de: iniciar } \\
\text { un proceso de ayuda por un periodo de tiempo estable, establecer } \\
\text { un contacto puntual o iniciar un proceso de derivación }\end{array}$ & $\begin{array}{lllll}1 & 2 & 3 & 4 & 5\end{array}$ & 12345 \\
\hline 29 & $\begin{array}{l}\text { Si la relación no tiene continuidad, finalizar de forma tal, que } \\
\text { la persona o familia pueda volver si lo necesita en un futuro } \\
\text { retomando la relación significativa que hemos establecido } \\
\text { (prevención) }\end{array}$ & 12345 & 12345 \\
\hline 30 & $\begin{array}{l}\text { Si se valora iniciar un proceso de ayuda estable por un tiempo, } \\
\text { identificar el tipo de contexto de intervención desde el cual se } \\
\text { inicia la relación de ayuda con la persona o familia (informativo, } \\
\text { asistencial, de consulta o asesoramiento, de mediación, clínico, } \\
\text { evaluativo, de control) }\end{array}$ & 123345 & 12345 \\
\hline 31 & $\begin{array}{l}\text { Manifestar interés por conocer cómo son las situaciones en que } \\
\text { la persona o familia se siente capaz de cambiar su situación de } \\
\text { dificultad y preguntar qué cosas hacen para que esto sea posible } \\
\text { (empowerment) y qué aspectos deberían cambiar para que la } \\
\text { situación planteada pueda mejorar (objetivos iniciales) }\end{array}$ & $\begin{array}{lllll}1 & 2 & 3 & 4 & 5\end{array}$ & 12345 \\
\hline
\end{tabular}




\begin{tabular}{|c|c|c|c|}
\hline & Criterios de realización & Importancia & Realización \\
\hline 32 & $\begin{array}{l}\text { Anclar el contexto de intervención profesional: al final del primer o } \\
\text { primeros encuentros con la familia o persona es importante dedicar } \\
\text { un tiempo para asegurarnos que el contexto inicial de intervención } \\
\text { profesional es claro para ambos, es decir, que la familia conoce cómo } \\
\text { trabajamos y que ambos, trabajador social y persona o familia, } \\
\text { tienen un propósito común en la relación de ayuda que se inicia }\end{array}$ & $\begin{array}{lllll}1 & 2 & 3 & 4 & 5\end{array}$ & 12345 \\
\hline 33 & $\begin{array}{l}\text { El acuerdo inicial se definirá de forma flexible, que permita durante } \\
\text { el proceso de estudio, ajustar o ampliar los acuerdos iniciales } \\
\text { (co-evolución del contexto), procurando que estén fundamentados } \\
\text { en una relación de cooperación, confianza y esperanza en que el } \\
\text { cambio es posible }\end{array}$ & $\begin{array}{lllll}1 & 2 & 3 & 4 & 5\end{array}$ & 12345 \\
\hline 34 & $\begin{array}{l}\text { Durante las entrevistas posteriores de estudio, el contexto inicialmente } \\
\text { acordado, puede ir evolucinando, dando lugar a un contexto } \\
\text { de intervención diferente al inicialmente acordado y por tanto, } \\
\text { introduciendo un nuevo procedimiento, una naturaleza de relación } \\
\text { diferente, una nueva manera de hacer (el cómo) } \\
\text { Acordar con la persona o familia que si durante el proceso de ayuda } \\
\text { formula nuevas demandas, el contexto de intervención profesional } \\
\text { podrá ir co-evolucionando. A medida que la relación es más } \\
\text { segura, la persona puede sentirse más confiada para hablar de otras } \\
\text { necesidades o problemas que pueden llevar a un nuevo acuerdo } \\
\text { sobre los contenidos a trabajar }\end{array}$ & 1232345 & 12345 \\
\hline 35 & $\begin{array}{l}\text { Si se ha valorado iniciar un proceso estable por un tiempo, acordar } \\
\text { un nuevo encuentro, consensuando los contenidos que vamos } \\
\text { a trabajar (para qué nos vamos a encontrar) de acuerdo con los } \\
\text { objetivos iniciales planteados y el contexto inicial de relación } \\
\text { profesional acordado }\end{array}$ & 122345 & 12345 \\
\hline 36 & $\begin{array}{l}\text { Al final de los encuentros, resumir-recapitular lo acontecido } \\
\text { durante el encuentro, destacando las necesidades expuestas, los } \\
\text { problemas planteados y las capacidades y puntos fuertes hallados, } \\
\text { consensuando y ajustando dicho resumen con la persona o los } \\
\text { miembros de la familia }\end{array}$ & $\begin{array}{lllll}1 & 2 & 3 & 4 & 5\end{array}$ & 12345 \\
\hline 37 & $\begin{array}{l}\text { Orientar la intervención a fortalecer, recuperar o mantener los } \\
\text { apoyos naturales de la persona o familia y en su defecto, llevar a } \\
\text { cabo intervenciones de poner en relación a la persona, potenciando } \\
\text { la creación de nuevas redes sociales }\end{array}$ & $\begin{array}{lllll}1 & 2 & 3 & 4 & 5\end{array}$ & 12345 \\
\hline 38 & $\begin{array}{l}\text { Establecer una relación de colaboración con otros profesionales } \\
\text { de otras áreas presentes en el caso (escolar, sanitaria, social, } \\
\text { judicial, psicológica, psiquiátrica...) desde una relación de } \\
\text { complementariedad que permita llegar a acuerdos de cooperación } \\
\text { profesional y con el consentimiento de la persona o familia }\end{array}$ & 122345 & 12345 \\
\hline 39 & $\begin{array}{l}\text { Afrontar las diferentes formas de ver un mismo caso en la red de } \\
\text { forma constructiva, mediante el uso de prácticas mediadoras y } \\
\text { buscando el consenso y la cooperación }\end{array}$ & $\begin{array}{lllll}1 & 2 & 3 & 4 & 5\end{array}$ & 12345 \\
\hline 40 & $\begin{array}{l}\text { Conocer el estado de salud física y mental del grupo familiar, su } \\
\text { grado de conciencia y de responsabilidad ante la enfermedad, así } \\
\text { como las consecuencias que acarrea en su dinámica cotidiana la } \\
\text { presencia de enfermedad/ discapacidad, mostrando interés por } \\
\text { conocer al cuidador. }\end{array}$ & 122345 & 12345 \\
\hline 41 & $\begin{array}{l}\text { Conocer la situación de la vivienda, si es un espacio que facilita } \\
\text { o entorpece la intimidad de los miembros (hacinamiento), si } \\
\text { el espacio tiene condiciones de habitabilidad (precariedad, } \\
\text { chabolismo), si está adaptada a las condiciones físicas de las } \\
\text { personas, si su ubicación permite el contacto con la comunidad }\end{array}$ & $\begin{array}{lllll}1 & 2 & 3 & 4 & 5\end{array}$ & 12345 \\
\hline
\end{tabular}




\begin{tabular}{|c|c|c|c|}
\hline & Criterios de realización & Importancia & Realización \\
\hline 42 & $\begin{array}{l}\text { Conocer la situación económica de la familia, determinar si las } \\
\text { necesidades básicas para la supervivencia y el desarrollo están } \\
\text { cubiertas. Conocer la organización del presupuesto familiar, el uso y } \\
\text { el valor que le dan al dinero }\end{array}$ & $1 \quad 23345$ & 12345 \\
\hline 43 & $\begin{array}{l}\text { Centrar el desarrollo de las entrevistas iniciales en la inclusión de } \\
\text { todos y en la comprensión de las razones personales que cada uno } \\
\text { manifiesta. (La entrevista no estará centrada en valorar los que } \\
\text { tienen o no razón, sino en comprender las razones de cada uno) }\end{array}$ & 1223345 & 12345 \\
\hline 44 & $\begin{array}{l}\text { Trasmitir verdadero interés no sólo en su problema sino también en } \\
\text { sus competencias y puntos fuertes como personas } \\
\text { (joining) }\end{array}$ & 1223345 & 12345 \\
\hline 45 & $\begin{array}{l}\text { Después de analizar la situación problema-necesidad desde las } \\
\text { diferentes áreas, evaluar si es oportuno otorgar el recurso solicitado } \\
\text { por la persona o familia de forma literal, si no lo es o si puede ser } \\
\text { contraproducente dada la situación estudiada }\end{array}$ & 122345 & 12345 \\
\hline 46 & $\begin{array}{l}\text { En caso necesario, re-definir la demanda con la finalidad de ofrecer } \\
\text { una propuesta que responda a la necesidad de la persona o familia }\end{array}$ & $\begin{array}{lllll}1 & 2 & 3 & 4 & 5\end{array}$ & 12345 \\
\hline 47 & $\begin{array}{l}\text { Prevenir el estancamiento y la cronicidad: Evaluar periódicamente } \\
\text { los cambios que puedan acontecer durante el proceso de estudio, } \\
\text { estableciendo relaciones entre las acciones, las propuestas, las } \\
\text { intervenciones, las actitudes del trabajador social y de otros } \\
\text { profesionales presentes en el caso, con las mejoras o los retrocesos } \\
\text { en la persona o familia ante la situación problema }\end{array}$ & 1223345 & 12345 \\
\hline 48 & $\begin{array}{l}\text { En el proceso de evaluación continua, valorar cuál es el grado de } \\
\text { satisfacción de la persona o familia sobre el proceso de ayuda que se } \\
\text { ha iniciado, así como los aspectos que le han resultado más útiles de } \\
\text { nuestro proceder }\end{array}$ & $\begin{array}{lllll}1 & 2 & 3 & 4 & 5\end{array}$ & 12345 \\
\hline 49 & $\begin{array}{l}\text { Ser consciente que la actitud } \\
\text { es fundamental para que el a } \\
\text { (auto-conocimiento) }\end{array}$ & 122345 & 12345 \\
\hline 50 & $\begin{array}{l}\text { Asegurar la confidencialidad como derecho de la persona o familia } \\
\text { y como deber del Trabajador Social con las únicas excepciones que } \\
\text { marca la ley, o en el caso de situaciones que representen un peligro } \\
\text { para sí mismo o un tercero }\end{array}$ & 122345 & 12345 \\
\hline 51 & $\begin{array}{l}\text { Asegurar la confidencialidad: en caso de trabajar un mismo caso } \\
\text { con diferentes servicios o proyectos, informar a la persona cliente } \\
\text { que los aspectos referidos a su proceso (avances y retrocesos) serán } \\
\text { compartidos con otros profesionales y que los detalles íntimos que } \\
\text { se puedan exponer en el sí de la relación de ayuda estarán sujetos a } \\
\text { la confidencialidad, a no ser que ambos valoren la importancia de } \\
\text { compartirlos y la persona autorice a ello }\end{array}$ & $\begin{array}{lllll}1 & 2 & 3 & 4 & 5\end{array}$ & 12345 \\
\hline 52 & $\begin{array}{l}\text { Activar el principio de individuación: reconocer y cor } \\
\text { singularidades de cada persona y familia }\end{array}$ & 1223345 & 12345 \\
\hline 53 & $\begin{array}{l}\text { Activar el principio de autodeterminación de la persona: estimular y } \\
\text { acompañar a las personas en el proceso de toma de decisiones y no } \\
\text { decidir por ellas }\end{array}$ & 1223345 & 12345 \\
\hline 54 & $\begin{array}{l}\text { Activar el principio de no enjuiciamiento: valorar con la persona } \\
\text { su situación con el objeto de establecer una relación de apoyo, } \\
\text { orientando, asesorando, informando... No se buscan culpables, sino } \\
\text { comprender junto con la familia las razones de sus actuaciones. Se } \\
\text { evitan las etiquetas que entorpecen la comprensión particular de } \\
\text { esta persona o familia }\end{array}$ & 1223345 & 12345 \\
\hline
\end{tabular}




\begin{tabular}{|c|c|c|c|}
\hline & Criterios de realización & Importancia & Realización \\
\hline 55 & $\begin{array}{l}\text { Activar el principio de aceptación de la persona o familia: aceptar } \\
\text { a la persona, respeto incondicional a la persona. No implica la } \\
\text { legitimación de conductas. Actitud profesional que garantiza la no } \\
\text { discriminación }\end{array}$ & $\begin{array}{lllll}1 & 2 & 3 & 4 & 5\end{array}$ & $\begin{array}{lllll}1 & 2 & 3 & 4 & 5\end{array}$ \\
\hline 56 & $\begin{array}{l}\text { Activar el principio de respeto a la persona: el Trabajador Social } \\
\text { de casos tendrá en cuanta las necesidades fundamentales de las } \\
\text { personas que solicitan ayuda (ser tratado como persona, no como } \\
\text { categoría profesional, poder expresar los sentimientos, ser aceptado, } \\
\text { encontrar comprensión y posibilidad de nuevas vías a su problema, } \\
\text { no verse juzgado, poder elegir por sí mismo sin recriminación, } \\
\text { conservar su integridad como persona y confiar en que toda la } \\
\text { información se mantendrá bajo la confidencialidad) }\end{array}$ & $\begin{array}{lllll}1 & 2 & 3 & 4 & 5\end{array}$ & $\begin{array}{lllll}1 & 2 & 3 & 4 & 5\end{array}$ \\
\hline 57 & $\begin{array}{l}\text { Evaluar periódicamente cómo llega a la persona o familia nuestra } \\
\text { actitud / estilo en la relación de ayuda: interesarse por saber cómo } \\
\text { se siente la persona después de cada entrevista, si su dignidad se } \\
\text { ha preservado, si se ha sentido respetado, qué cosas de nuestro } \\
\text { procedimiento le alientan en la búsqueda de soluciones, le infunden } \\
\text { confianza y esperanza }\end{array}$ & $\begin{array}{lllll}1 & 2 & 3 & 4 & 5\end{array}$ & $\begin{array}{lllll}1 & 2 & 3 & 4 & 5\end{array}$ \\
\hline 58 & $\begin{array}{l}\text { harco de relación claro en } \\
\text { ación y con un propósito }\end{array}$ & $\begin{array}{lllll}1 & 2 & 3 & 4 & 5\end{array}$ & $\begin{array}{lllll}1 & 2 & 3 & 4 & 5\end{array}$ \\
\hline 59 & $\begin{array}{l}\text { En aquellos casos en que se valora que el caso va a necesitar una } \\
\text { relación de ayuda estable por un tiempo, construir una alianza de } \\
\text { ayuda en los primeros encuentros con la persona o familia para } \\
\text { propiciar un buen resultado en la intervención }\end{array}$ & 1223345 & 122345 \\
\hline 60 & $\begin{array}{l}\text { Genera } \\
\text { cambio }\end{array}$ & 1223345 & $\begin{array}{lllll}1 & 2 & 3 & 4 & 5\end{array}$ \\
\hline 61 & $\begin{array}{l}\text { Generar enganche en el proceso de ayuda: la persona y el Trabajador } \\
\text { Social ven un sentido al trabajo iniciado, los objetivos y las tareas } \\
\text { pueden negociarse entre ambos. El cambio es vivido como posible } \\
\text { por ambos }\end{array}$ & $\begin{array}{lllll}1 & 2 & 3 & 4 & 5\end{array}$ & $\begin{array}{lllll}1 & 2 & 3 & 4 & 5\end{array}$ \\
\hline 62 & de es & $\begin{array}{lllll}1 & 2 & 3 & 4 & 5\end{array}$ & $\begin{array}{lllll}1 & 2 & 3 & 4 & 5\end{array}$ \\
\hline 63 & $\begin{array}{l}\text { Generar segurida } \\
\text { capacidad a la pe } \\
\text { para facilitar la a }\end{array}$ & 1223045 & $\begin{array}{lllll}1 & 2 & 3 & 4 & 5\end{array}$ \\
\hline 64 & $\begin{array}{l}\text { Construir la relación de a } \\
\text { la persona o familia, difer } \\
\text { creen y lo que sienten }\end{array}$ & $\begin{array}{lllll}1 & 2 & 3 & 4 & 5\end{array}$ & $\begin{array}{lllll}1 & 2 & 3 & 4 & 5\end{array}$ \\
\hline 65 & $\begin{array}{l}\text { Construir un acuerdo de intervención para el cambio después de } \\
\text { un proceso de estudio-comprensión que haya permitido conocer, } \\
\text { comprender y consensuar cuál es la situación problema así como los } \\
\text { recursos y capacidades que le impiden y/o facilitan hacerse cargo de } \\
\text { la situación: Construir un compromiso mutuo de trabajo } \\
\text { (co-diagnóstico) }\end{array}$ & 1123345 & $\begin{array}{lllll}1 & 2 & 3 & 4 & 5\end{array}$ \\
\hline 66 & $\begin{array}{l}\text { Comprender la situación en sus diversas dimensiones y disponer de } \\
\text { una primera valoración contrastada y consensuada con la persona o } \\
\text { familia, antes de intervenir } \\
\text { (co-diagnóstico) }\end{array}$ & $1 \begin{array}{lllll}1 & 2 & 3 & 4 & 5\end{array}$ & $\begin{array}{lllll}1 & 2 & 3 & 4 & 5\end{array}$ \\
\hline
\end{tabular}




\begin{tabular}{|c|c|c|c|}
\hline & Criterios de realización & Importancia & Realización \\
\hline 67 & $\begin{array}{l}\text { Conjuntamente con la persona o familia, identificar las áreas } \\
\text { relacionadas con el problema (laboral, relacional, económica, salud, } \\
\text { vivienda...) y definir los objetivos de cambio teniendo en cuenta la } \\
\text { disponibilidad de los recursos existentes } \\
\text { (co-diagnóstico) }\end{array}$ & 122345 & 12345 \\
\hline 68 & $\begin{array}{l}\text { Conjuntamente con la persona o familia, concretar las áreas en que } \\
\text { valora necesaria nuestra ayuda } \\
\text { (co-diagnóstico) }\end{array}$ & 1223445 & 12345 \\
\hline 69 & $\begin{array}{l}\text { Si es necesario, ayudar a la persona o familia a identificar las cosas } \\
\text { que tienen que cambiar para que la situación mejore (co-diagnóstico) }\end{array}$ & $\begin{array}{lllll}1 & 2 & 3 & 4 & 5\end{array}$ & 12345 \\
\hline 70 & $\begin{array}{l}\text { Definir objetivos que sean alcanzables, concretados en unidades } \\
\text { pequeñas, expresados de forma conductual y relacional, expresados } \\
\text { en términos positivos y evaluables en un tiempo determinado } \\
\text { (co-diagnóstico) }\end{array}$ & 122345 & 1223045 \\
\hline 71 & $\begin{array}{l}\text { Definir los objetivos de forma que la responsabilidad y la } \\
\text { competencia en los logros sean atribuidas a la persona /familia } \\
\text { (co-diagnóstico) }\end{array}$ & 122345 & $\begin{array}{lllll}1 & 2 & 3 & 4 & 5\end{array}$ \\
\hline 72 & $\begin{array}{l}\text { Transmitir esperanza: ayudar a la persona o familia a visionarse en } \\
\text { un futuro donde el cambio ha sido posible } \\
\text { (co-diagnóstico) }\end{array}$ & 122345 & 1223445 \\
\hline 73 & $\begin{array}{l}\text { Una vez finalizado el proceso de estudio-comprensión y haber } \\
\text { compartido y ajustado con la persona o familia el co-diagnóstico } \\
\text { Concretar con la persona o familia, los elementos que definen el } \\
\text { contexto de intervención profesional para el cambio: chequear si han } \\
\text { quedado claros los temas o asuntos que juntos hemos acordado } \\
\text { que necesitaban mejorar o cambiar (objetivos y metas) y compartir } \\
\text { con la familia cómo solemos trabajar cuando la relación de ayuda } \\
\text { va dirigida a introducir cambios o mejoras relacionados con este } \\
\text { tipo de temas o asuntos. Es decir, explicar y consensuar con } \\
\text { la familia el método que vamos a utilizar en este momento de } \\
\text { intervención hacia el cambio, las características que tendrán los } \\
\text { encuentros y nuestra relación, los espacios y los tiempos que vamos } \\
\text { a utilizar, los requisitos del servicio para iniciar dicho proceso, la } \\
\text { confidencialidad, la intensidad, los límites y el calado del trabajo } \\
\text { que vamos a iniciar } \\
\text { Según el tipo de problema, el contexto de intervención para el cambio } \\
\text { desde el cual se encauzará la relación de ayuda, comportará una } \\
\text { naturaleza de relación diferente y un método diferenciado. }\end{array}$ & 1223345 & 123345 \\
\hline 74 & $\begin{array}{l}\text { Manifestar interés para obtener de la persona o familia un feedback } \\
\text { final sobre el encuentro en concreto o el proceso que hemos } \\
\text { iniciado: ¿creen que el encuentro que hemos compartido les ha sido } \\
\text { útil?, ¿creen que avanzamos en la consecución de objetivos?, ¿viven } \\
\text { que ambos compartimos el mismo propósito en la relación de } \\
\text { ayuda?, ¿viven que pueden expresar sus opiniones y que estas son } \\
\text { valiosas para nosotros? }\end{array}$ & 123345 & 12345 \\
\hline 75 & Auto-evaluar la intervención y planificar el nuevo encuentro & 123345 & 123345 \\
\hline
\end{tabular}




\section{Bibliografía}

Anderson, H. (1997). Conversation, Language, and Possibilities: A Postmodern Approach to Therapy. New York: Basic Books. Edición castellana: Anderson, H. (2000). Conversación, lenguaje y posibilidades: un enfoque postmoderno de la terapia. Buenos Aires: Amorrotu.

Andolfi, M. (2000). Il colloquio relazionale. Roma: Accademia di Psicotepia della Famiglia. Edición castellana: Andolfi, M. (2003). El coloquio relacional. Barcelona: Paidós.

Aponte, H., (1976). Underorganization in the poor family (pp. 432-448). In P.J. Guerin, Jr. (ed.), Family therapy: Theory and practice. New York: Gardner Press.

Azpeitia, B., Bezunartea, P., Malagón. S., Maya,A., Orts, M., Pérez, D. y Utrilla, M. (2003). Manual para el Trabajo Social de Acompañamiento en los itinerarios de inserción. Madrid: Colegio Oficial de Trabajadores Sociales de Madrid.

BAteson, G. (1999). Steps to an Ecology of Mind. Chicago: Chicago University Press. Edición en castellano: Bateson, G. (2013).Pasos hacia una ecología de la mente. Buenos Aires: Lumen.

CAmpanini, A. y LupPi, F. (1991). Servicio social y modelo sistémico. Barcelona: Paidós.

CARDONA, J. (2008). Inventario para el análisis de la relación de ayuda entre el Trabajador social y el Cliente durante la fase de estudio y evaluación de la situación-problema. NA. Registral 00/2008/5206. RCPI. Madrid: Ministerio de Cultura.

CARDONA, J. y CAMPOS, J. F. (2009). Cómo determinar un contexto de intervención: Inventario para el análisis de la relación de ayuda entre el Trabajador/a Social y el Cliente durante la fase de estudio y evaluación de la situación problema. Portularia, IX, 2, 17-35.

CARdona, J. (2012). La definición del contexto de intervención en el trabajo social de casos. Tesis doctoral, Universidad de las Islas Baleares. Recuperado en dfts. uib.cat/digitalAssets/286/286359_Cardona_Cardona_Josefa.pdf.

Cardona-Cardona, J., Montaño-Moreno, J.-J., y Campos-Vidal, J. F. (2016). Definition of Intervention Contexts in Social Casework Practice: Predictor Variables. British Journal of Social Work. First published online January 12, 2016. doi:10.1093/bjsw/bcv142.

CARPENTER, J y TREACHER, A.(1989). Marital and familiar therapy. Oxford: Basil Blacwell. Edición en castellano: Carpenter, J. y Treacher, A. (1993). Problemas y soluciones en terapia familiar y de pareja. Barcelona: Paidós.

Cirillo, S. (1994). El cambio en contextos no terapéuticos. Barcelona: Paidós.

DE SHAZER, S. (1988). Clues: Investigating solutions in brief therapy. New York: W.W. Norton. Edición en castellano: de Shazer, S. (1990). Claves para la solución en terapia breve. Barcelona: Gedisa. 
DE ShAZER,S. (2009). Claves en Psicoterapia Breve: una teoría de la solución. Barcelona: Gedisa.

D’ADDA, L. y GAllione, C. (1983). Contesti e richieste di prestazione nella pratica del Servizio Sociale. Prospettive Sociali e Sanitarie 21, pp. 7-11.

ESCUDERO, V. y LÓPEZ, S. (2003). Familia, Evaluación e Intervención. Madrid: Editorial CCS.

FEInTEIn, AR. The evaluation of validity. En: Feinstein,AR. Clinimetrics. New Haven (CT): Yale University Press, 1987.

Friedlander, M., Escudero, V. y HeAtherington, L. (2009). La alianza terapéutica. Barcelona: Paidós.

Hamilton, G. (1940). Theory and Practice of Social Work. New York: Columbia University Press. Edición en castellano: Hamilton, G. (1960). Teoría y Práctica del Trabajo Social de Casos. México: Prensa Médica Mexicana.

Hollis, F. (1972,). Casework: A Psychosocial Therapy. New York: Random House. (Second edition).

IMBRE-BlaCK, E. (1989). Families and Larger Systems: A Family Therapist's Guide Through the Labyrinth. New York: Guilford. Edición en castellano: Imbre-Black, E (2001). Familias y sistemas amplios. El terapeuta familiar en su laberinto. Buenos Aires: Amorrortu.

Ituarte Tellaeche, A, (1992). Procedimiento y proceso en el Trabajo Social Clínico. Madrid: Siglo XXI.

LAMAS, C. (1997). Los primeros contactos. En Coletti, M. y Linares, J. (Comp). La intervención sistémica en los servicios sociales ante la familia multiproblemática. Barcelona: Paidós.

LIPCHIK, E. (2002). Beyond technique in solution-focused therapy: Working with emotions and the therapeutic relationship. New York: The Guilford Press. Edición en castellano: Lipchik, E. (2004). Terapia centrada en soluciones. Más allá de la técnica. Buenos Aires: Amorrortu.

MinuChin,S. (1984). Familias y Terapia Familiar. Barcelona: Paidós.

MinuChIn,S. y FISHMAN, H.(1981). Family Therapy Techniques. Cambridge: Harvard University Press. Edición castellana: Minuchin, S. y Fishman, H.(1984). Técnicas de terapia familiar. Barcelona: Paidós.

Perlman, H. (1960). Social Casework. Chicago: Chicago University Press. Edición en castellano: Perlman, H.H. (1970). El trabajo social individualizado. Madrid: Rialp.

O’Hanlon, B. y Weiner-Davis,M. (1989). In Search of Solutions: A New Direction in Psychotherapy. New York: Norton. Edición en castellano: O`Hanlon, B. y Weiner-Davis,M. (1990). En busca de soluciones: un nuevo enfoque en psicoterapia. Barcelona: Paidós.

O’HAnlon,B. y Bertolino, B.(1999). Invitation To Possibility Land: An Intensive Teaching Seminar With Bill O’Hanlon. Routledge: PA. Edición en castellano: 
O`Hanlon, B. y Bertolino, B. (2001). Desarrollar posibilidades. Barcelona: Paidós.

Ripoll-Millet, A. (2001). Familias, Trabajo Social y Mediación. Barcelona: Paidós. Rossell, T. (1989). La entrevista en el Trabajo Social. Barcelona: Euge.

Rosemberg, M. (2006). Comunicación no violenta: un lenguaje de vida. Camarones,

Ar.: Gran Aldea Editores.

SATIR, V. (1986). Psicoterapia familiar conjunta. México: Prensa Médica Mexicana. Selekman, M. (1993). Pathways to Change. New York: The Guilford Press. Edición en castellano: Selekman, M. (1996). Abrir caminos para el cambio. Barcelona: Gedisa.

TUERLinCKX, J. (1973). La asistencia social individualizada, $3^{a}$ Edición. Madrid: Aguilar.

Watzlawick, P., Weakland, J. y Fish, R. (1976). Change: Principles of problem formation and resolution. New York: Norton. Edición castellana: Watzlawick, P., Weakland, J. y Fish, R. (1989). Cambio. Formación y solución de los problemas humanos. Barcelona: Herder.

White, M. (1993). Medios narrativos para fines terapéuticos. Barcelona: Paidós.

White, M. (2002). El enfoque narrativo en la experiencia de los terapeutas. Barcelona: Gedisa.

White, M. (2007). Maps of narrative practice. New York: Norton. 